BRX TH-6308

CALT-TH 2016-026

\title{
One-loop gravity divergences in $D>4$ cannot all be removed
}

\author{
S. Deser \\ Walter Burke Institute for Theoretical Physics, \\ California Institute of Technology, Pasadena, CA 91125; \\ Physics Department, Brandeis University, Waltham, MA 02454 \\ deser@brandeis . edu
}

\begin{abstract}
Unlike the one-loop QG divergences in $D=4$, which can all be transformed away, those in arbitrary higher (even) $D$ cannot.
\end{abstract}

\section{$1 \quad D=4$}

Explicit loop calculations in quantum gravity (QG) originated over four decades ago with 'tHooft and Veltman's [1] one-loop results in 4D. They noted that, even without calculation, the one-loop, hence quadratic in curvature, leading correction of the source-free theory could always be removed: First, the Riemann-squared term can be turned into a sum of Ricci- and scalar- curvature-squared ones using the pure divergence nature of the 4D Gauss-Bonnet (GB) invariant,

$$
\int d^{4} x \epsilon^{\alpha \beta \gamma \delta} \epsilon^{\rho \sigma \mu \nu} R_{\alpha \beta \rho \sigma} R_{\gamma \delta \mu \nu} \sim \int d^{4} x\left[R_{\mu \nu \rho \sigma} R^{\mu \nu \rho \sigma}-4 R_{\mu \nu} R^{\mu \nu}+R^{2}\right] \sim 0 .
$$

Second, the remaining, $\int R_{\mu \nu}^{2}$ and $\int R^{2}$, terms can then be relegated to higher order by the field redefinition

$$
\delta g_{\mu \nu}=a(g, R) R_{\mu \nu}+b(g, R) g_{\mu \nu} R,
$$

one that is clearly available in all $D$ to remove monomials involving at least one Ricci tensor or scalar. [It is essential that the quadratic corrections are, by definition, NOT to be taken as part of the "kinetic" term, which remains $R$, and of course cannot be removed.]

The development of string theory and its corrections to QG in $D=10$ has made the study of higher $D$ relevant (see [2] for a recent example). We note here, in the same no-calculation spirit, that the no-correction result breaks down in $D=2 n>4$, because there is now more than one independent monomial in $\left(R_{\mu \nu \alpha \beta}\right)^{n}$, while there is still only one, GB $(D>4)$, available to remove them. Even-parity counterterms (and GB) cannot exist at all in odd dimensions, since they would have to have an odd number of derivatives, hence of indices: Power counting in GR trivially establishes that all one-loop counterterms are of $D$-derivative order because vertices/propagators 
are universally of $+2 /-2$ derivative order and cancel each other when adding a new vertex, so that the cutoff dependence is just that of the integration dimension, $\int d^{D} p$, for any number of external gravitons.

\section{$2 \quad D>4$}

The relevant GB invariant in any even, $D=2 n,(1)$ simply generalizes to

$$
G B(D=2 n)=\int d^{2 n} x \epsilon^{\cdots} \epsilon^{\cdots}\left(R_{\ldots}^{1} \quad R_{\ldots}^{n}\right) \sim 0
$$

while of course (2) still removes all terms involving at least one Ricci or curvature scalar in any $D$. Consider $D=6$ for concreteness; there, counterterms and GB are of 6 -derivative order. The candidate one-loop invariants are then of three types,

$$
\int \text { Riem }^{3}, \quad \int \text { Riem } d d \text { Riem, } \quad G B(6),
$$

( $d$ denotes a covariant derivative), plus (harmless) terms containing at least one Ricci or $R$ factor. The single GB can only convert one of the two independent Riem ${ }^{3}$ into the "Ricci" type. The $\int$ Riem $d d$ Riem can be reduced to Riem $^{3}$ form by the cyclic Bianchi identities $R_{\mu \nu[\alpha \beta ; \rho]}=0$, div Riem $=$ curl Ricci, to remove the $d d$ in favor of a possible extra Riem where use of $[d, d] \sim$ Riem is needed. The same conclusions apply, a fortiori, to any higher $D$; a detailed list of curvature monomials for any $D$ can be found in [3]. A separate interesting set of actions, the Weyl invariant ones, also increases with dimension, beyond the unique $\int C^{2}$ of $4 D$; a sample is given in [4], there being two $\int C^{3}$ (and one $\int C \square C$ ) in 6D: there are now multiple Weyl "gravities".

\section{Comments}

We close with some conceivable, if unlikely, possible exceptions to these no-calculation statements. First, although there is no obvious selection principle that would allow only the one GB (Riem $)^{n}$ counterterm (3) from among the many possible ones at higher dimensions, this should, and can, easily be checked explicitly by using the methods of [1]. Second, the catalog of [3] lists the locally independent monomials; there might be some integral relations among them - that is, under the integral sign, they may differ from each other by divergences and terms involving Riccis. This too seems unlikely (except for the $\int \operatorname{Riem}^{p} \square^{q} \operatorname{Riem}^{n-p-q}$ type above), simply because $4 \mathrm{D}$ has the special property that, to leading, $\sim h_{\mu \nu}^{2}$, order, all momenta are sandwiched between just two $h$, so on-Einstein shell where $0=\operatorname{Ricci}=(p p h)_{\mu \nu}$, there is only one $\left(h p^{4} h\right) \sim(\operatorname{Riem})^{2}$ monomial. At higher $D$, the leading terms are now of the form $h p p \ldots h p p \ldots h \ldots$; the $p$ and $h$ indices, are more "segregated", which accounts for the increase of $\int$ Riem $^{n}$ combinations. 


\section{Acknowledgements.}

I thank J. Franklin for Latexerity. This work was supported by grants NSF PHY-1266107 and DOE \# desc0011632.

\section{References}

[1] G. 'tHooft, M.J.G. Veltman, One loop divergencies in the theory of gravitation, Ann.Inst.H.Poincare Phys.Theor. A20 (1974) 69.

[2] Clifford Cheung, Grant N. Remmen, Positivity of Curvature-Squared Corrections in Gravity, arXiv:1608.02942.

[3] S.A. Fulling et al., Normal forms for tensor polynomials.1: The Riemann tensor, Class.Quant.Grav. 9 (1992) 1151.

[4] S. Deser, A. Schwimmer, Geometric classification of conformal anomalies in arbitrary dimensions, Phys.Lett. B309 (1993) 279-284, arXiv/9302047. 